\title{
ANATOMICAL FEATURES
}

\section{OF THE}

LUMBO

SACRAL

SPINE

By R. TREVOR-JONES

An interesting collection of abnormal lumbar vertebrae and sacra are housed in the Department of Anatomy, Medical School, University of Cape Town. This material inspired me to investigate the musculo-skeletal anatomy, as seen in the dissecting room, in an attempt to explain certain aspects of backache. Clinical records of the subjects dissected were too brief for any correlation of findings and symptoms.

The lumbo-dorsal fascia varies in thickness and over the sacrum blends with the fascial origin of the erector spinae muscle. From the erector spinae aponeurosis inter-muscular septa pass forwards to separate Multifidus completely from the sacro spinalis group of muscles.

- Multifidus arises from the dorsum of the sacrum, the posterior medial edge of the iliac crest between - posterior superior and posterior inferior iliac spines, the deep surface of the combined lumbo-dorsal and erector spinae fascia and the medial surface of the intermuscular septum which separates it from Longissimus. The intermuscular septum, in its upwards extension is attached to the inferior aspect of the mammillary processes while Multifidus arises from the superior aspect as well as the inter articular joint capsules.

Careful dissection reveals that Multifidus can be separated into sagittal lamellae arranged segmentally although the muscle looks like a solid wedge. That portion medial to the posterior inferior iliac spine passes to the sacrum and lower four lumbar vertebrae while the portion from the posterior superior iliac spine passes to first lumbar and twelfth thoracic vertebrae.

Longissimus and Iliocostalis have a common origin just lateral to the posterior superior iliac spine, from the erector spine, from the erector spinae fascia and from the lateral surface of the intermuscular septum separating it from Multifidus. It can be divided into coronally placed musculotendonous lamellae passing to the accessory tubercles and adjacent laminae of the lumbar vertebrae.

Ilio-costalis is separated from Longissimus by a neurovascular plane and an intermuscular septum. This muscle also can be separated into coronally placed lamellae passing to the transverse processes of the lumbar vertebrae. The outer portions of these lamella are formed into encircling loops which, with the erector spinae fascia and lumbar fascia firmly enclose the back muscles in the lumbo-sacral region.

This segmental pattern is found in foetuses. Multifidus then has sacral segments. A six piece foetal sacrum was associated with incomplete muscle segmentation. This lack of muscle differentiation was also found in an adult cadaver with a sacralisation of the fifth lumbar vertebra on the left side. The muscle anomaly however occurred on the 
right side. In this case too, it was noted that the descending branch of the fourth lumbar nerve was stretched and angulated over the enlarged transverse process of the fifth lumbar vertebra.

In partial sacralisations then it would appear that backache could be due to either the pain of the pseudarthrosis or direct nerve involvement.

Disc degeneration has been adequately described by other workers. However, other soft tissue features were noted.

In fat subjects, intermuscular deposits of fat are found between the muscle segments, around the apophyseal joints and large extra articular deposits on the posterior aspects of the superior articular bearing facets of the sacrum, the fifth and fourth lumbar vertebrae (an accumulation of fat in front of the sacrum, on superior and inferior surfaces of the pelvic diaphragm could be of gynaecological importance).

Degenerations of the interspinous ligaments were frequently noted. These are frequently associated with joint like cavities between closely approximated spinous processes.

The apophyseal joints vary considerably in shape, size and inclination. The articular facets are seldom symmetrical and even in fresh specimens do not articulate accurately. Asymmetry of articular facets is often associated with osteoarthritic changes of vertebral bodies and apophyseal joints.

The apophyseal joint capsules are usually thickened inferiorly and extend backwards to blend with the inter- spinous ligaments. A. meniscoidal intra-articular synovial fringe encompasses the periphery of the intra-articular facets. In osteoarthritis of the apophyseal joints this synovial fringe enlarges so that in severely affected joints it extends as a ragged curtain across the joint. Nipping of this fringe could possibly account for sudden spasms of backache.

Enlargement of the intra- and extra- vertebral venous plexuses is often found at osteo-arthritic levels of the vertebral column as a whole. This may well be related to diminished intervertebral movement. The intervertebral foramen transmits the nerve root, two small arteries and three or more venous connections between intra- and extravertebral venous plexuses. Its boundaries consist of intervertebral disc and adjacent vertebral bodies anteriorly, the superior vertebral notch inferiorly, the larger inferior vertebral notch superiorly and the ligamentum flavum covering the apophyseal joint posteriorly. Loose connective tissue and fat surrounds the nerves, arteries and veins. Intervertebral movement affects the size and shape of the intervertebral foramen. In flexion all diameters of the foramen are increased while extension caused a diminution of these diameters. Intervertebral movement obviously subjects the emerging veins to alterations of shape and pressure and conceivably causes a pumping effect assisting venous return.

These features suggest that adequate intervertebral movement is essential for a pain free back. Clinical experience shows that backache is most frequent among office workers, housewives and the less active individuals. In contrast the African population is generally free of backache and in fact only suffer from backache when "Westernised". 